The abstracts are only available online, free of charge, under www.karger.com/doi/10.1159/000350375

Regional North American Annual Meeting of the World Federation of Neurology Research Group on Neuroepidemiology

University of California at San Diego, San Diego, Calif., March 22, 2013

\title{
Program and Abstracts
}

Guest Editors

Marc G. Weisskopf, Boston, Mass.

Amethyst Leimpeter, Oakland, Calif.

Stephen K. Van Den Eeden, Oakland, Calif. 


\section{Program}

Abstract No.

\begin{tabular}{|c|c|c|}
\hline 08:00 AM & Registration Opens & \\
\hline 08:30 AM & Poster Set up and Viewing & P1-P3 \\
\hline 09:00 & Introduction & \\
\hline $09: 15$ & $\begin{array}{l}\text { Cognitive Function and Risk of Parkinson's Disease in the Cardiovascular Health Study } \\
\text { T.G. Ton }\end{array}$ & 1 \\
\hline $09: 35$ & $\begin{array}{l}\text { Cumulative Exposure to Lead and Cognition in Persons with Parkinson's Disease } \\
\text { M.G. Weisskopf }\end{array}$ & 2 \\
\hline 09:55 & $\begin{array}{l}\text { Non-Motor Subtypes of Early Parkinson Disease in the Parkinson's Progression } \\
\text { Markers Initiative } \\
\text { S. Jain }\end{array}$ & 3 \\
\hline $10: 15$ & Break & \\
\hline $10: 30$ & $\begin{array}{l}\text { Physical Trauma and Risk of ALS: A Population-Based Study } \\
\text { R. Seals }\end{array}$ & 4 \\
\hline $10: 50$ & $\begin{array}{l}\text { Multicenter ALS Cohort Study of Oxidative Stress and Disease Progression: Structure } \\
\text { and Methodology } \\
\text { H. Mitsumoto }\end{array}$ & 5 \\
\hline $11: 10$ & $\begin{array}{l}\text { Environmental Predictors of Early and Late Onset Amyotrophic Lateral Sclerosis } \\
\text { G. Logroscino }\end{array}$ & 6 \\
\hline $11: 30$ & Business Meeting & \\
\hline $11: 45$ & Lunch & \\
\hline $12: 15$ & Poster Viewing & P1-P3 \\
\hline $12: 30$ & $\begin{array}{l}\text { Keynote Address - The Millennium Cohort Study. An Epidemiologic Assessment of } \\
\text { Mental Health Outcomes among Returning Service Members } \\
\text { Nancy F. Crum-Cianflone }\end{array}$ & \\
\hline 01:30 & $\begin{array}{l}\text { Endotoxin Exposure and Parkinsonism among Women Textile Workers in Shanghai, } \\
\text { China: Preliminary Findings } \\
\text { H. Checkoway }\end{array}$ & 7 \\
\hline $01: 50$ & $\begin{array}{l}\text { Blood Lead Concentrations in Essential Tremor - An Epidemiological Study in } \\
\text { Diyarbakir, Turkey } \\
\text { O. Dogu }\end{array}$ & 8 \\
\hline
\end{tabular}


02:10 Nodding Syndrome in W. Equatoria, South Sudan: Environmental, Nutritional and Infectious Factors

P. Spencer

02:30 Final Closing and Adjournment

\section{Poster Presentations}

Heart Rate Variability as a Potential Indicator of Prodromal Parkinson's Disease

P1

S.W. Cash

Body Composition and the Risk of Parkinson Disease in the Cardiovascular Health Study

P2

S. Jain

Amyotrophic Lateral Sclerosis (ALS) Disease Trajectory Is Similar in Bulbar to Respiratory and P3

Vice-Versa

B. Rix Brooks 


\section{Abstracts}

Neuro

epidemiology

\section{Oral Presentations}

01

\section{Cognitive Function and Risk of Parkinson's Disease in the Cardiovascular Health Study}

\author{
T.G. Ton, L. Jie, M.L. Biggs, J.A. Delaney, D. Landsittel, S. Hu, \\ L. Curtis, S. Searles Nielsen, W.T. Longstreth, H. Checkoway, S. Jain \\ Seattle, Wash., Durham, N.C., and Pittsburgh, Pa., USA
}

Objective: We explored the association between cognitive function and risk of Parkinson's disease (PD). Background: Cognitive deficits have been documented in patients with PD but whether decreased cognitive function precedes PD diagnosis is unknown. Methods: The Cardiovascular Health Study enrolled 5,888 adults aged 65 years and older in the US. Cognitive function was measured at baseline using the digit symbol substitution test (DSST) and the 100-point modified mini-mental state (3MS) examination. Higher scores indicate better function in both tests. We identified PD from 1989 through 2007 using self-report, medications, inpatient ICD-9 codes, medical charts, and Medicare information including outpatient services, skilled nursing homes, hospice, home healthcare, and type of physician submitting the claim. Two movement disorders specialists adjudicated final PD status. We assessed the association between performance on DSST and 3MS and PD risk using multivariate Cox regression models. Results: We identified 165 incident cases of PD over 70,796 person-years of follow-up. After adjusting for age, gender, race, education, smoking, alcohol consumption, depression and diabetes, higher DSST score was associated with a decreased risk of PD (HR $=0.97 ; 95 \%$ CI: 0.96, 0.99). Those in the highest DSST quartile were substantially less likely to develop PD ( $\mathrm{HR}=0.53,95 \% \mathrm{CI}: 0.30,0.94)$. The HR for the highest vs. lowest quartile for $3 \mathrm{MS}$ was 0.73 (95\% CI: $0.40,1.30)$, although this association was not statistically significant. In secondary analyses that excluded incident PD within 5 years of baseline, continuous DSST score remained inversely associated with PD risk $(\mathrm{p}=0.03)$. Conclusion: Deficits in processing speed and perhaps cognitive function precede $\mathrm{PD}$ diagnosis, but it remains unknown whether these are etiologic factors themselves, manifestations of a shared etiology, or early pre-motor indicators of PD.

\section{2 \\ Cumulative Exposure to Lead and Cognition in Persons with Parkinson's Disease}

\author{
J. Weuve, D.Z. Press, F. Grodstein, R.O. Wright, H. Hu, M.G. Weisskopf \\ Boston, Mass., New York, N.Y., USA and Toronto, Ont., Canada
}

Objective: To evaluate the association between cumulative lead exposure and cognitive function in Parkinson's Disease (PD). Background: Dementia is an important consequence of Parkinson's disease (PD), with few known modifiable risk factors. Cumulative exposure to lead, at levels experienced in the community, may exacerbate PD-related neural dysfunction, resulting in impaired cognition. Methods: We evaluated cumulative lead exposure, gauged by tibia and patella bone lead concentrations, among 101 persons with PD ('cases') and, separately, 50 persons without PD ('controls'). We assessed cognitive function using a telephone battery developed and validated in a separate sample of PD patients. We used linear regression techniques to estimate the association between bone lead and cognitive function adjusted for potential confounders. We assessed the interaction between lead and case-control status by including a multiplicative interaction term between the two in the model. Results: After multivariable adjustment, higher tibia bone lead concentration among PD cases was associated with worse performance on all of the individual telephone tests. In particular, tibia lead levels corresponded to significantly worse performance on a telephone analog of the Mini-Mental State Examination and tests of working memory and attention. Moreover, higher tibia bone lead concentration was associated with significantly worse global composite score encompassing all the cognitive tests $(\mathrm{P}=0.04)$. The magnitude of association per standard deviation increment in tibia bone lead level was equivalent to the difference in global scores among controls in our study, who were approximately 7 years apart in age. The tibia lead-cognition association was notably stronger within cases than within controls $(\mathrm{P}($ difference $)=0.06)$. Patella bone lead concentration was not consistently associated with performance on the tests. Conclusions: These data provide evidence suggesting that cumulative exposure to lead may result in worsened cognition among persons with $\mathrm{PD}$. 


\section{3 \\ Non-Motor Subtypes of Early Parkinson Disease in the Parkinson's Progression Markers Initiative}

\section{S. Jain, S. Young Park}

Pittsburgh, Pa., USA

Objective: To explore whether subtypes of Parkinson disease (PD) may be defined by non-motor features in a cohort of recently diagnosed PD patients. Background: Parkinson disease (PD) is a multi-system, multi-organ disease with varied clinical presentations and pathologic changes in the central nervous system and peripheral end-organs. The clinical period commences with a premotor phase characterized by varied non-motor features follow by motor signs that lead to diagnosis. As non-motor features predate motor signs, characterizing patterns of non-motor features in early PD my inform models of pathogenesis and disease surveillance. Methods: The Parkinson's Progression Markers Initiative is an observational cohort which currently contains 345 individuals with PD who at enrollment were: at least 30 years old; diagnosed within that last 2 years; and not treated for PD. Baseline assessments of motor, neurobehavioral, cognitive, autonomic, sleep and olfactory features were completed. K-means cluster analysis was performed using baseline non-motor characteristics. The number of clusters was determined by comparing the sum of squared error (SSE) distance between each member of a cluster and the cluster center in actual data versus 250 randomized versions. To plot clusters, principal components analyses were performed. Clusters were validated by ANOVA and Fischer's exact test on variables not used for clustering. Results: Four PD subtypes derived from nonmotor features emerged (\% of PD population): (1) Cognitive and Olfactory Impairment (17\%) with the most impaired cognitive and olfactory function; (2) Younger onset, mildest non-motor features, slowest progression (32\%); (3) Intermediate burden (38\%); and (4) Non-cognitive, non-motor impairment with rapid progression (13\%) with the most severe sleep, depressive and autonomic symptoms and highest prevalence of impulsive/compulsive features. Conclusions: These preliminary results suggest PD subtypes defined by non-motor features exist early in the clinical period. Such patterns of non-motor features may be present prior to diagnostic motor signs in longitudinal cohorts with incident PD cases.

\section{4 \\ Physical Trauma and Risk of ALS: A Population- Based Study}

\author{
R. Seals, J. Hansen, O. Gredal, M. Weisskopf \\ Boston, Mass., USA, Copenhagen, and Aarhus, Denmark
}

Objective: To assess the relationship between physical trauma and risk of ALS in Denmark. Background: Clinical observations and case-control studies have indicated that physical trauma could be a risk factor for ALS; however, many of these studies were small and relied on self-report of trauma history. We conducted the larg- est and most comprehensive registry-based study of trauma and ALS. Methods: Our study base is the entire population of Denmark from the years 1982 to 2009 . From this population we selected all 3,650 incident ALS cases as documented in the Danish Hospital Registry, and a random sample of 100:1 age- and sex-matched controls from the Danish Central Person Registry. Trauma history from 1977 was assessed from the Danish National Hospital Registry, and each trauma was classified into one of six classes (open wounds, fractures, dislocations, nerve injuries, contusion and crushing, concussions). Prior trauma for each was considered dichotomously, both unlagged and lagged five years. Results: A history of any prior trauma was significantly associated with ALS $(\mathrm{OR}=1.31 ; 95 \% \mathrm{CI} 1.00-1.26)$. On 5 yr-lagged analysis, however, a history of any prior trauma was not associated with ALS (OR = $1.01 ; 95 \%$ CI $0.92-1.11$ ). The effect of prior trauma is confined to the 365 days prior to ALS diagnosis. None of the specific traumas lagged five years showed any significant association with ALS risk, although dislocations were borderline associated $(\mathrm{OR}=1.14$; 95\% CI 0.97-1.34). Conclusions: This national nested case-control study is the largest study of hospital admission for trauma and risk of subsequent ALS. Results are consistent with earlier results from smaller studies suggesting that the observed increase in ALS risk in the year following a trauma is driven by either reverse causation or over-ascertainment. There is little evidence that history of hospital-diagnosed concussion elevates ALS risk. These results apply only to trauma resulting in hospitalization.

\section{5 \\ Multicenter ALS Cohort Study of Oxidative Stress and Disease Progression: Structure and Methodology}

\author{
H. Mitsumoto, P. Factor-Litvak, H. Andrews, R. Goetz, J. Hupf, \\ J. Singleton, C. Campanella
}

New York, N.Y., USA

Background: ALS is a devastating, rapidly fatal neurodegenerative disease of a largely unknown cause. It is clinically characterized by progressive skeletal muscle paralysis and eventual respiratory failure. The mean survival is approximately 40 months following symptom onset, however the survival time is highly variable. No prospective investigations have attempted to determine factors which either accelerate or decelerate disease progression. Recent clinical and epidemiological studies of ALS suggest diverse environmental and lifestyle risk factors, many of which are related to the generation of oxidative stress (OS). We hypothesize that these extrinsic factors act throughout the disease course, are associated with varying levels of OS and thus influence disease progression. Methods: To test this hypothesis, we recruited 350 newly diagnosed sporadic ALS patients from 15 ALS centers across the US. Exposure to OS is measured via questionnaire and biomarkers. ALS progression is determined with the ALSFRS-R and other clinical features every 3 to 6 months up to 24 months or death. At baseline and follow-up, we obtained OS biomarkers (urinary isoprostane and 8-oxodeoxyguanosine) and lipid profiles, and we 
measured current and past environmental, psychological, dietary, and lifestyle factors associated with OS. Additional stored specimens include DNA, plasma and skin biopsies. Results: Statistical analyses will examine associations between OS biomarkers, lipid profiles and an empirically derived OS index based on questionnaire data and ALS progression. The OS index will include a variety of occupational, environmental, lifestyle, and psychological factors. To our knowledge, this is the first prospective, interdisciplinary, in-depth multicenter epidemiological investigation of OS related to ALS progression. Conclusion: Our project will increase the understanding of the mechanisms involved in disease prognosis and may be the first step toward new treatment to target multiple OS sites in ALS. Baseline enrollment has been completed, and we are currently following the cohort.

\section{6}

\section{Environmental Predictors of Early and Late Onset Amyotrophic Lateral Sclerosis}

\author{
P. Messina, M. Bandettini di Poggio, A. Chiò, M. Filosto, \\ G. Logroscino, C. Lunetta, J. Mandrioli, F. Pisa, E. Vitelli, E. Beghi \\ Milano, Genova, Torino, Brescia, Bari, Modena, Udine and Lodi, \\ Italy
}

Objective: To compare the history of environmental exposure of early onset and late onset Amyotrophic Lateral Sclerosis (ALS) patients. Background: ALS is a fatal neurodegenerative disease with high phenotypic variability. Differences could be explained by several genetics and environmental factors. Methods: Eligible cases were definite, probable or possible ALS patients with an age at diagnosis $<55$ years (Early ALS, EA) or $>74$ years (Late ALS, LA). Each case was matched to a hospital control for age, sex and residency (Early and Late controls EC, LC). All participants were asked to fill a structured questionnaire which contained demographic data, history of environmental exposures and dietary habits. Results: 81 EA, 71 LA, 64 EC and 62 LC were recruited. A conditional logistic model (ALS vs. controls) accounted for 126 patients (64 Early and 62 Late) and 126 controls. Independent risk factors included history of lead gasoline exposure, Adj. OR $(95 \% \mathrm{CI})=5.7(1.2-26.6)$, low intake of vegetables, Adj. OR $(95 \%$ $\mathrm{CI})=8.4(2.5-28.1)$ and history of foods (putatively) containing glutamate, Adj. OR $(95 \% \mathrm{CI})=2.4(1.0-5.7)$. Comparing the four groups (EA, EC, LA, LC) EA patients reported (in each comparison) a significantly higher percentage of individuals exposed to lead $(21.9 \%, 9.4 \%(\mathrm{p}=0.05), 8.1 \%(\mathrm{p}=0.03), 8.1 \%(\mathrm{p}=0.03))$, solvents $(32.8 \%, 14.1 \%(\mathrm{p}=0.01), 9.7 \%(\mathrm{p}=0.00), 6.5 \%(\mathrm{p}=0.00))$ and lead gasoline $(19.4 \%, 4.8 \%(\mathrm{p}=0.01), 6.5 \%(\mathrm{p}=0.03), 3.2 \%$ $(\mathrm{p}=0.01))$. Conclusions: Our results suggest that some environmental factors may contribute to accelerate ALS onset. However, recall bias is an alternative explanation.
07

\section{Endotoxin Exposure and Parkinsonism among Women Textile Workers in Shanghai, China: Preliminary Findings}

H. Checkoway, W. Li, C.M. Tanner, S.C. Hu, X. Wang, D.L. Gao, W. Fan, J. Ding, J. Hu, R.M. Ray, S. Searles Nielsen, D.B. Thomas

Seattle, Wash. and Sunnyvale, Calif., USA, and Shanghai, China

Objective: To test the hypothesis that cotton textile workplace exposure to endotoxin is etiologically related to parkinsonism (PS) prevalence and severity in a dose-dependent manner. Background: Endotoxin (lipopolysaccharide [LPS]) is a product of gram negative bacteria, and a widespread environmental contaminant, including in cotton dust. There is a convincing animal model of LPS-induced PS in rodents. Methods: From a previously established cohort of women textile workers in Shanghai, China, we recruited 569 retired women, aged 50-87, previously employed in cotton textile factories and an age-matched non-exposed reference group of 298 retired women textile workers from non-cotton textile factories. Approximately 97 percent of participants were never smokers. Study subjects' cumulative occupational exposure to endotoxin (endotoxin units $/ \mathrm{mg}^{3} \mathrm{x}$ years) were available from previous research on cancer risks in this cohort. Study neurologists conducted a standardized neurological examination, including the Unified Parkinson Disease Rating Scale Motor Section (UPDRS3). Results: Based on initial exam findings, and applying a provisional case definition of UPDRS3 score $>15$, we identified 32 (5.6\%) PS cases among exposed and 16 (5.4\%) cases among non-exposed reference workers. Ongoing analysis involves identifying subjects with possible exclusionary criteria (e.g. history of stroke), refining the clinical case definition, and estimation of the exposure-response trend. These findings will be presented at the WFN meeting. Conclusion: The observed pattern of crude crosssectional results does not indicate an etiologic relation between PS and cumulative occupational endotoxin exposure in Shanghai women textile workers. However, this conclusion may change as findings from more refined analyses emerge.

\section{8 \\ Blood Lead Concentrations in Essential Tremor - An Epidemiological Study in Diyarbakir, Turkey}

T. Tanrikulu, Y. Tamam, L. Tamer Gumus, O. Dogu

Diyarbakir and Mersin, Turkey

Objective: The aim of this study was to determine blood lead concentrations in patients with ET and healthy controls. Background: Two previous clinical and epidemiological studies showed increased blood lead levels in ET patients. Methods: 253 ET cases over 50 years of age ascertained from a population-based study in the province of Diyarbakır in Turkey, were matched with 261 controls from the same population. All patients and controls were examined by study neurologists. Results: Age and other de- 
mographic variables of the ET cases and the participants in the control group were similar. Mean $( \pm S D)$ blood lead concentration for ET cases was $4.23 \pm 1.7 \mu \mathrm{g} / \mathrm{dL}$ (range $=2.03-13.3 \mu \mathrm{g} / \mathrm{dL}$ ), and $2.5 \pm 1.1 \mu \mathrm{g} / \mathrm{dL}$ (range $=0.2-4.5 \mu \mathrm{g} / \mathrm{dL}$ ) for the controls. In the unadjusted logistic regression model, blood lead concentration is correlated with diagnosis (ET case as against control): $\mathrm{OR}=7.32$; $95 \%$ CI, 4.95-10.81; $p<0.001$. A correlation was found between severity of tremor (total tremor score) and blood lead concentration $(\mathrm{r}=0.09, \mathrm{p}<0.03)$ in all sampling. The correlation between blood lead concentration and duration of tremor in ET cases was not at a significant level $(\mathrm{r}=0.71 ; \mathrm{p}=0.23)$, especially after adjustment for age $(r=0.85 ; p=0.37)$. Conclusions: These findings are similar to those of the two previous studies, and show a correlation between lead, a toxic material in the environment, and ET, a widespread neurological condition.

\section{9}

Nodding Syndrome in W. Equatoria, South Sudan: Environmental, Nutritional and Infectious Factors

\author{
P. Spencer, K. Vandemaele, M. Richer, V.S. Palmer, S. Chungong, \\ M. Anker, Y. Ayana, M.L. Opoka Douglas, N. Klaucke, A. Quarello, \\ J.K. Tumwine
}

Geneva, Switzerland, Juba, South Sudan, Portland, Oreg., Brasstown, N.C. and Amherst, Mass., USA, Cairo, Egypt, Padova, Italy, and Kampala, Uganda

Background: Nodding Syndrome is an idiopathic seizure disorder of children in sub-Saharan Africa. The disorder is reported to be spreading in South Sudan and northern Uganda. Objective: To describe environmental, nutritional, infectious, and other factors that existed before and during the de novo 1991 appearance and subsequent increase in cases through 2001 in then-southern Sudan. Methods: Household surveys, informant interviews, and case-control studies conducted in Lui town and Amadi village in 2001-2002 were supplemented in 2012 by informant interviews in Lui and Juba. Results: Nodding Syndrome was associated with Onchocerca volvulus and Mansonella perstans infections, with food use of a variety of sorghum (serena) introduced as part of an emergency relief program, and inversely associated with a history of measles infection. There was no evidence to suggest exposure to a manmade neurotoxic pollutant or chemical agent, other than chemically dressed seed intended for planting but used for food. Food use of cyanogenic plants was documented, and exposure to fungal contaminants could not be excluded. Conclusion: Nodding Syndrome in South Sudan has an unknown etiology. Further research is recommended on the association of Nodding Syndrome with onchocerciasis/mansonelliasis and neurotoxins in plant materials used for food.

\section{Poster Presentations}

\section{P1 \\ Heart Rate Variability as a Potential Indicator of Prodromal Parkinson's Disease}

S.W. Cash, S.M. Goldman, S.S. Sullivan, G.S. Bhudhikanok, K. Comyns, C. Meng, L. Rees, C.M. Tanner, J.W. Langston

Sunnyvale, and Palo Alto, Calif., USA

Objective: Evaluate the potential utility of heart rate variability (HRV) in identifying prodromal Parkinson's disease (PD) by investigating HRV in persons with PD, idiopathic REM sleep behavior disorder (iRBD), and controls. Background: PD likely develops many years prior to the onset of motor symptoms, providing a long latent period for intervention if detected early. A large proportion of $\mathrm{iRBD}$ patients develop $\mathrm{PD}$, and thus may be considered to have prodromal PD. Cardiac autonomic dysfunction (CAD) may be an early manifestation of $\mathrm{PD}$ and has been reported in $\mathrm{R} B \mathrm{DD}$. HRV derived from an EKG is a non-invasive measure of $\mathrm{CAD}$, and could potentially serve as a prodromal PD indicator. Methods: PD patients $(\mathrm{n}=12)$, polysomnographically verified iRBD patients $(n=4)$, and healthy controls $(n=9)$ underwent a 5-minute resting EKG. RR intervals were extracted, ectopic beats removed, and HRV measures calculated using Kubios v.2.0 software. Pairwise group differences were assessed using nonparametric Mann-Whitney U tests. Trends across all three groups were assessed using nonparametric Jonckheere-Terpstra tests with exact p-values. Results: SDHR and RRTRI (time-domain measures) were significantly lower in PD cases than controls $(\mathrm{p}=0.009$ and $\mathrm{p}=0.034$, respectively). Significant frequency-domain differences were observed for $\operatorname{VLF}\left(\mathrm{ms}^{2}\right)(\mathrm{p}=0.012), \operatorname{LF}\left(\mathrm{ms}^{2}\right)(\mathrm{p}=0.003)$, total power $\left(\mathrm{ms}^{2}\right)(\mathrm{p}=0.018), \operatorname{LF}(\mathrm{nu})(\mathrm{p}=0.028), \operatorname{HF}(\mathrm{nu})(\mathrm{p}=0.028)$, and $\mathrm{LF} / \mathrm{HF}(\mathrm{p}=0.028)$. Trends across $\mathrm{PD}, \mathrm{iRBD}$, and control groups were seen for SDHR $(\mathrm{p}=0.006), \operatorname{RRTRI}(\mathrm{p}=0.024), \operatorname{VLF}\left(\mathrm{ms}^{2}\right)(\mathrm{p}=$ $0.015), \mathrm{LF}\left(\mathrm{ms}^{2}\right)(\mathrm{p}=0.003)$, total power $\left(\mathrm{ms}^{2}\right)(\mathrm{p}=0.011), \mathrm{LF}(\mathrm{nu})$ $(\mathrm{p}=0.018), \operatorname{HF}(\mathrm{nu})(\mathrm{p}=0.018)$, and LF/HF $(\mathrm{p}=0.018)$. Age- and gender-adjusted logistic regression models yielded similar results. Conclusions: Most HRV measures differed for PD patients compared to controls, signifying cardiac dysautonomia. Significant trends across groups were observed for several HRV measures, with $\mathrm{iRBD}$ patient values intermediate between controls and PD patients, consistent with the hypothesis that many iRBD patients have prodromal PD. EKG-derived HRV could prove useful in identifying prodromal PD. 


\section{P2}

\section{Body Composition and the Risk of Parkinson Disease in the Cardiovascular Health Study}

S. Jain, M.L. Biggs, P. Gibbs, D. Landsittel, L. Curtis, S. Hu, W.T. Longstreth, T.G. Ton

Pittsburgh, PA., Seattle, Wash. and Durham, N.C., USA

Objective: To explore the association between body composition and the risk of being diagnosed with Parkinson disease (PD). Background: Weight loss (possibly due to loss of fat mass) is common is PD. The enteric nervous system is among the earliest regions to demonstrate neuropathology, which may result in changes in body composition. Methods: The Cardiovascular Health Study (CHS) is a prospective community-based cohort of 5,888 adults aged 65 years and older. Body mass index (BMI) and bioelectrical impedance resistance (BIR) were measured at baseline. PD cases from 1989-2007 were adjudicated using self-report, anti-parkinsonian medications, medical records, and Medicare billing claims. The index date was the earliest date of evidence for PD from any source. Association between risk of PD and BIR, or measures derived from BIR (fat mass, \% body fat, lean body mass and total body water) were assessed using Cox regression models controlling for potential confounding factors (age, sex, smoking, race, education, alcohol consumption, physical activity, and caffeine intake). Results: 165 incident PD cases were identified over 70,796 person-years of follow-up. In fully adjusted models, only the highest quartile of BIR $(>611 \Omega)$ was associated with lower risk of PD (HR:0.50, 95\% CI:0.27-0.94), and point estimates with fat mass were similar. Excluding PD cases within 5 years of baseline, this association was near significance $(p<0.06)$. Conclusions: High BIR (presumably due to high fat mass) may be associated with lower risk of PD. The lack of significant associations with other BIR derived measures in fully adjusted models may be due to insufficient power. The marginal significance after excluding cases within 5 years of baseline suggests that either high BIR is an etiologic factor or marker of latent disease. Further investigation in larger studies is needed.

\section{P3 \\ Amyotrophic Lateral Sclerosis (ALS) Disease Trajectory Is Similar in Bulbar to Respiratory and Vice-Versa}

B. Rix Brooks

Charlotte, N.C., USA

Background: Gastrostomy tube (GT) placement and initiation of non-invasive ventilation (NIV) are employed to improve nutrition and respiration in patients with ALS. Either intervention occurs at different times and each constitutes a definite milestone in the ALS Dashboard staging system being evaluated to assess domain (bulbar, respiratory) stage specific disease progression. Objectives: Compare disease trajectory for patients with ALS who receive GT before NIV or NIV before GT in a single-center-based clinical audit. Methods: From 2007-2012, 35 patients [19 F;16 $\mathrm{M}$ ] received GT before NIV and 46 patients $24 \mathrm{M} ; 22 \mathrm{~F}$ ] received NIV before GT. Disease trajectory was assessed by ALSFRS-R and ALS Dashboard. Statistical analysis was performed with MedcCalc software(version 12. 2.1.0). Results: Time from GT to NIV in 35 patients with ALS who had bulbar disease domain progression first (mean $=9.6$ months; $95 \% \mathrm{CI}=5.9-13.8$ months) was identical to time from NIV to GT in 46 patients with ALS who had respiratory disease domain progression first (mean $=9.8$ months; $95 \% \mathrm{CI}=$ 6.1-14.4 months). Overall survival in each group was comparable regardless of the initial intervention. Conclusions: Deployment of GT first in bulbar domain progressing patients followed by NIV or NIV first in respiratory domain progressing patients followed by GT proceeds along similar disease trajectories.

Discussion: ALS Dashboard change within bulbar and respiratory domains provides comparable rates of disease trajectory change over time. Further studies will be required to assess whether newer diagnostic techniques identifying pharmacological or physical treatment interventions will affect these milestone changes in a similar fashion. 


\section{Author Index}

Neuro epidemiology

Andrews, H. 300

Anker, M. 302

Ayana, Y. 302

Bandettini di Poggio, M. 301

Beghi, E. 301

Bhudhikanok, G.S. 302

Biggs, M.L. 299, 303

Campanella, C. 300

Cash, S.W. 302

Checkoway, H. 299, 301

Chiò, A. 301

Chungong, S. 302

Comyns, K. 302

Curtis, L. 299, 303

Delaney, J.A. 299

Ding, J. 301

Dogu, O. 301

Factor-Litvak, P. 300

Fan, W. 301

Filosto, M. 301

Gao, D.L. 301

Gibbs, P. 303

Goetz, R. 300

Goldman, S.M. 302

Gredal, O. 300

Grodstein, F. 299

Numbers refer to page numbers

Hansen, J. 300

Hu, H. 299

$\mathrm{Hu}, \mathrm{J} .301$

Hu, S. 299, 303

$\mathrm{Hu}$, S.C. 301

Hupf, J. 300

Jain, S. 299, 300, 303

Jie, L. 299

Klaucke, N. 302

Landsittel, D. 299, 303

Langston, J.W. 302

Li, W. 301

Logroscino, G. 301

Longstreth, W.T. 299, 303

Lunetta, C. 301

Mandrioli, J. 301

Meng, C. 302

Messina, P. 301

Mitsumoto, H. 300

Opoka Douglas, M.L. 302

Palmer, V.S. 302

Pisa, F. 301

Press, D.Z. 299

Quarello, A. 302
Ray, R.M. 301

Rees, L. 302

Richer, M. 302

Rix Brooks, B. 303

Seals, R. 300

Searles Nielsen, S. 299, 301

Singleton, J. 300

Spencer, P. 302

Sullivan, S.S. 302

Tamam, Y. 301

Tamer Gumus, L. 301

Tanner, C.M. 301, 302

Tanrikulu, T. 301

Thomas, D.B. 301

Ton, T.G. 299, 303

Tumwine, J.K. 302

Vandemaele, K. 302

Vitelli, E. 301

Wang, X. 301

Weisskopf, M. 300

Weisskopf, M.G. 299

Weuve, J. 299

Wright, R.O. 299

Young Park, S. 300 